\title{
Les plans de gestion des crises bancaires Une tautologie ou une utopie?
}

\section{Résumé :}

La crise financière de 2008 a mis en exergue les insuffisances des outils et des pratiques de gestion des crises bancaires dans le monde, c'est pourquoi les autorités de réglementation, de surveillance et de supervision bancaire ont décidé de mettre en place de nouveaux instruments pour: faire face aux frasques des établissements bancaires et des institutions financières d'importance systémique (Systemically Important Financial Institutions - SIFIs), en renforçant la qualité et le niveau de leur fonds propres afin d'améliorer la couverture du risque ; réduire le coût phénoménal de l'aléa moral qui anime l'activité de l'ensemble du système bancaire et dont les effets se ressentent jusqu'à l'économie réelle. À travers cette analyse, nous essayons de démontrer que malgré les réformes élaborées afin de contenir les effets négatifs résultants des défaillances des SIFIs, le risque de voir se transformer une crise bancaire en crise systémique n'est pas encore écarté.

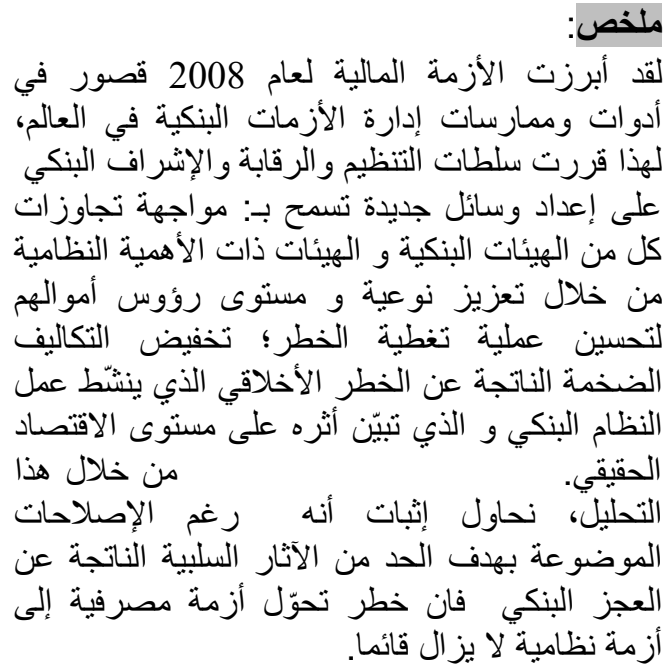

Benelmouffok Souheila

Département sciences de Gestion Université Constantine2

\section{Introduction :}

$\mathrm{Au}$ cours des trois dernières décennies, le renforcement de la réglementation et de la surveillance des banques actives à l'international avait constitué un axe prioritaire des efforts visant à assurer la stabilité financière dans le monde, en réduisant la probabilité de faillite des institutions financières de grandes envergures, étant donné que, leurs défaillances pourraient avoir de graves répercussions non seulement sur l'économie nationale mais aussi sur l'économie mondiale. La récente crise financière $\mathrm{a}$ 
démontré que malgré une réglementation et une surveillance rigoureuse, le risque de faillite ne peut être éliminé et la faillite de la Lehman Brothers une banque d'investissement vieille de plus de 150 ans l'atteste. Cet effondrement avait constitué un véritable séisme financier planétaire en raison de l'intégration financière internationale et au comportement tous azimuts des investisseurs sur les marchés.

Cette connectivité du système financier dans notre monde globalisé ne permettait pas aux Etats de laisser faillir plus d'établissements financiers, car la contagion aurait était désastreuse pour l'économie réelle. À cet effet, des montants considérables de fonds publics ont été mis à la disposition des banques pour soutenir leur activité, mais par la suite, les gouvernements ont clairement indiqué qu'un tel soutien ne pourra pas être renouvelé, vu que, ces aides publiques avaient pesé lourdement sur les contribuables, mais aussi, avaient alourdi la dette publique des Etats, marquant ainsi la fin du « too big to fail » qui signifie : trop gros pour tomber.

Dès lors, les instances tant nationales qu'internationales ont consacré beaucoup d'efforts à la conception d'un régime, qui permet de mieux maîtriser les faillites bancaires et donc de maintenir l'efficience du système financier. Alors :

Comment est le nouveau paradigme du sauvetage des banques ?

Comment peut-on maintenir à flot le système bancaire en période de crise sans avoir recours au fonds publics?

Comment peut-on palier le coût épouvantable des dépassements du secteur bancaire?

Quels sont les instruments qui permettent la stabilisation financière?

Avant de procéder au décorticage de ces questions, passant en revue cette crise qui a fait naître la tourmente économique, financière, politique, populaire... laissant derrière elle que scepticisme et pessimisme.

\section{I- Synthèse de la crise}

\section{1- La genèse de la crise}

Au début des années 2000 la Réserve fédérale américaine (Fed) exerça une politique monétaire accommodante qui s'est traduit par des taux directeurs très bas, afin d'éviter une récession aux Etats-Unis suite à : l'éclatement de la bulle internet en Mars 2000 et au recul du secteur des nouvelles technologies qui a suivi; les attentats du 11 Septembre 2001 et leurs répercussions sur l'économie. Par ailleurs, dans le cadre de la Community Reinvestment Act $(\mathrm{CRA})^{1}$, le gouvernement américain a mis en place une politique d'accession à la propriété qui encourageait les banques à accorder des prêts hypothécaires à des ménages peu solvables, l'idée était de permettre aux emprunteurs à revenus modestes de contracter un emprunt immobilier dit «Subprime », moyennant un taux d'intérêt fixe et promotionnel durant les premières années du prêt, mais qui devient par la suite variable et indexé sur le taux directeur de la Fed, le tout majoré d'une prime de risque très élevée afin de compenser le niveau de risque supérieur à la moyenne supporté par les banques.

Simultanément, les banques américaines se sont mises à prendre des risques excessifs en prêtant moins attention à la qualité des crédits accordés, visiblement exhortées par l'accroissement incessant du prix de l'immobilieri ${ }^{2}$ ostensiblement rassurées par le filet de sécurité financière et par un montage financier complexe portant le nom de «titrisation", celui-ci leur permettait de se refinancer tout en réduisant le risque inhérent à leur activité en transformant des créances - le plus souvent à risque - en titres cotés au maximum (AAA), avec la complicité des agences de notation (Moody's, Standard \& Poor's, Fitch Ratings) qui tiraient une partie essentielle de leur revenu de l'activité de conseiller qu'elles proposaient aux banques pour les aider à mettre au point et à noter de tels titres.

Ces titres à court terme et à taux bas étaient émis et vendus par des structures de financement - mises en place au préalable par les banques - appelées Special Purpose Vehicle (SPV) à des investisseurs à travers le monde entier, qui cherchaient à accroître leurs rendements 
dans des produits rémunérateurs. Majoritairement, ces investisseurs étaient des fonds d'investissement, soit appartenant à ces mêmes banques génératrices de ces montages financiers, soit finançaient par elles. Sinon, c'était des Organismes de Placement Collectif en Valeurs Mobilières (OPCVM), ou encore d'autre banques du système bancaire ; malencontreusement, tous ignoraient que ces titres recelés des niveaux de risque très élevés.

Le déclenchement de la crise des subprimes

Durant les années 2004-2006 la Fed décida de relever progressivement son taux directeur (de $1 \%$ à 5,25\%) afin de réduire les pressions inflationnistes grandissantes aux EtatsUnis ; en plus, à partir de 2006 la courbe des prix de l'immobilier subissait une forte régression dans plusieurs régions des Etats-Unis en raison de la baisse de la demande, ceux-ci entraînèrent un surenchérissement des mensualités de remboursement des crédits immobiliers à taux variables, de sorte que, de nombreux foyers américains étaient en situation de défaut de paiement. Subséquemment, il eut un afflux de saisie et de vente de la part des établissements de crédit pour les biens portés en garantie lors de l'emprunt, provoquant une chute vertigineuse des prix, puis un déséquilibre du marché immobilier et au final son effondrement total, astreignant ainsi les établissements à récupérer que partiellement la somme prêtée.

Le cumul des pertes de valeur supportés par le système bancaire depuis le premier semestre de l'année 2006; l'ensemble des dépassements pratiqués par les différents établissements de crédit depuis quelques années et surtout le laxisme permis depuis le début des années 2000 par le gouvernement américain ont été les causes principales du déclenchement de la crise des subprimes aux Etats-Unis révélée au grand jour dès l'été 2007, une crise que tout le monde croyait cantonner aux prêts hypothécaires américains, donc aux Amériques mais, qui au final renfermait de futurs événements sinistres à l'échelle mondiale.

\section{L'enchainement des tribulations}

Dès l'apparition de la crise des subprimes les tribulations sont allées en crescendo : pour commencer les agences de notation abaissaient la note des titres issus des montages financiers, car ils étaient susceptibles de contenir des créances à risque voir même des prêts subprimes, cet acte a fait germer des suspicions chez les investisseurs - qui croyaient avoir investis dans des titres sûrs et peu risqués - ce qui les a poussés à écouler leurs investissements rapidement. Seulement, ce désencombrement a déprécié leur prix, en raison de la méthode de valorisation de ces titres qui suit les nouvelles règles comptables internationales, à savoir : déterminer selon leur coût du moment (valorisation à la juste valeur), cette chute excessive des prix avait entraîné la baisse des indices boursiers, en même temps que, l'effondrement des marchés financiers. De ce fait, une nouvelle crise a été causée (i.e. Une crise boursière).

Postérieurement, de nouvelle tensions se sont manifestées en raison des déclarations de faillites de certains établissements de crédit, devenus insolvables à cause de leur investissement dans les titres à risque en période d'euphorie ; ces faillites en chaîne avaient créé un mouvement de panique chez les épargnants qui se sont ruées vers leur banque afin de retirer leur patrimoine, cette situation déclencha une crise de confiance au niveau des marchés interbancaires car, les banques en excès de capitaux ne voulaient plus prêter à celles en déficit, provoquant une envolée des taux directeur interbancaire et de la prime de risque de contrepartie, ce qui s'est reflété ultérieurement par une crise de liquidité.

$\mathrm{Au}$ final, cette crise de liquidité déboucha sur une crise bancaire qui de suite avait amorcé une dynamique sélective de la part des banques et des marchés financiers, occasionnant une raréfaction progressive des crédits à travers le monde entier, puis une crise de crédit. Ce nouveau marasme accrut le ralentissement économique dans plusieurs pays, face à cette quasi banqueroute il s'est imposé aux banques centrales, à la banque centrale européenne et surtout à la Réserve fédérale américaine (Fed) de procéder à de massives injections de liquidité - à plusieurs reprises - sur le marché interbancaire afin de permettre aux établissements bancaires de 
refinancer leur activité, de doper l'économie et surtout d'éviter le déclenchement d'une crise systémique.

Cependant, ces sauvetages in extremis n'étaient pas toujours un front succès car beaucoup de banques continuaient à être en situation d'insolvabilité, dans le but d'endiguer une possible fermeture généralisée, des mesures d'aide supplémentaires ont été prises par certains Etats tels que: la nationalisation de la Northern Rock par le gouvernement britannique travailliste; le placement sous tutelle du gouvernement américain des deux géants du crédit hypothécaire (Fannie Mae et Freddie Mac) ; le rachat des engloutis par des concurrents en meilleure situation à l'instar de la Merrill lynch qui a été rachetée par sa concurrente Bank of America ; d'autre malheureusement n'ont pas eu cette chance comme la Lehman Brothers une banque d'investissement multinationale qui fit faillite le 15 Septembre 2008.

Malencontreusement, les interventions des Etats pour le sauvetage des banques provoquèrent une nouvelle asphyxie (i.e. Une crise de la dette publique), du fait que, les finances publiques ont été lourdement sollicitées pour résoudre cette énorme crise financière, dont les répercussions étaient insupportables et gravissimes: fermeture en chaîne des entreprises, licenciement en masse des travailleurs, augmentation du taux de chômage, une croissance en berne, population tourmentée et désolation généralisée. Afin de redresser leur finance publique, réduire leur déficit et relancer la croissance, nombreux pays ont été contraint de suivre une politique de rigueur qui prône la hausse de la fiscalité et la baisse des dépenses publiques.

Cette synthèse met en exergue un truisme irrécusable : c'est la désinvolte dans laquelle immerge le système bancaire qui est à l'origine de cette crise financière. Néanmoins, est ce que toutes les institutions financières composant le système bancaire qui sont mises en causes dans cette crise?

Manifestement "non", ceux sont les institutions financières dites d'importances systémiques (IFIS) (Systemically Important Financial Institutions - SIFIs) qui gangrènent le système bancaire. Perçues comme ne devant pas faillir, du fait de: leur taille, leur interdépendance, leur complexité, leur manque de substituabilité et leur envergure mondiale, ces institutions bénéficient d'une protection implicite de la part de leur Etat.

Alors, jusqu'où et à quand le public peut-il et doit-il supporté les dépassements du secteur bancaire ? À quand les réformes qui protègent les contribuables ?

Compte tenu du coût économique et financier des interventions des Etats, de l'augmentation du risque subjectif (aléa moral) associé à l'industrie bancaire, il était devenu nécessaire de prendre des mesures supplémentaires pour réduire les externalités négatives associées à la défaillance d'établissements financiers d'importance systémique mondiale.

Avant d'évoquer ces mesures, il faut d'abord déterminer les institutions financières d'importances systémiques, mais aussi, l'aléa moral qui guide leur activité depuis maintenant une vingtaine d'années.

\section{Les institutions financières d'importance systémique}

Dans le cadre de Bâle III et du Dodd-Frank Act $^{3}$ le terme employé pour désigner les institutions financières d'importance systémique (Systemically Important Financial Institutions SIFIs) est : banques systémiques.

Une banque est dite systémique si sa faillite aurait des externalités négatives qui, si elles ne sont pas traitées, peuvent déstabiliser le système financier et avoir un impact négatif sur l'économie réelle. (Thomson, 2010)

Les ministres de l'économie du G20 se sont accordés sur des critères d'identification des institutions et des marchés d'importance systémique en Octobre 2009, sur la base des propositions conjointes du Fonds Monétaire International (FMI), de la Banque de Règlement International (BRI) et du Conseil de Stabilité Financière (CSF).

Les trois principaux critères retenus sont ${ }^{4}$ : 
La taille : il s'agit d'apprécier le volume des services financiers fournis par une entité individuelle ou un groupe. La taille au sens de l'identification du risque systémique est une notion exhaustive et recouvre les expositions, autrement dit les risques en bilan et hors bilan de l'entité observée.

La substituabilité : il s'agit d'apprécier la dépendance relative du système financier aux services financiers fournis par une entité individuelle, afin d'apprécier la résilience du système à la disparition de cette entité.

L'interconnexion: il s'agit d'apprécier les liens directs et indirects entre institutions financières qui vont faciliter la propagation du risque systémique et sa contagion à l'économie réelle.

Le Conseil de Stabilité Financière a identifié la liste des banques dont le risque est considéré comme systémique.

Cette liste est représentée dans le tableau figurant sur l'annexe 1.

La défaillance ou les difficultés financières auxquels se sont exposés ces grandes institutions financières d'envergure mondiale lors de la crise financière en 2008 ont provoqué des chocs dans tout le système financier, qui se sont répercuté implacablement sur l'économie réelle.

En réaction à la crise, des mesures supplémentaires ont été mise en place par les autorités de contrôle bancaire afin de réduire la gravité des problèmes crées par la défaillance des établissements financiers d'importance systémique, parmi ces mesures ${ }^{5}$ :

Renforcer la qualité et le niveau des fonds propres au sein du système bancaire afin d'améliorer la couverture des risques.

Instaurer un ratio de levier destiné à soutenir le régime fondé sur les risques.

Constituer des volants de conservation de fonds propres et contra-cycliques.

Instaurer une norme internationale de liquidité.

\section{L'aléa moral (le risque subjectif)}

L'aléa moral (moral hazard, en anglais) est le risque de perte encouru par un prêteur, en raison d'événements imprévisibles et/ou des imperfections du marché. Ce risque est lié à la moralité de l'individu et à sa situation personnelle plutôt qu'à la nature même de l'opération ${ }^{6}$.

Quelles sont les causes qui ont conduit à la propagation de l'aléa moral dans l'industrie bancaire?

\section{L'augmentation du volume des transactions financières :}

En raison de la libération des mouvements de capitaux au cours des années 80 et du développement technologique, les activités financières ont littéralement explosé.

L'augmentation de l'effet de levier du système financier :

La réglementation impose aux banques de détenir un pourcentage minimum de fonds propres par rapport au risque encourus, mais afin d'échapper à cette règle (qui limite l'effet de levier) nombre d'institutions ont eu recours à diverses méthodes :

La titrisation : un modèle anglo-saxon qui signifie « initier des prêts puis les distribuer aussitôt à des investisseurs sous formes de titres » autrement dit (Originate and distribute).

La création de « véhicule hors bilan » a permis à de nombreuses banques de constituer des actifs non soumis à des règles aussi strictes que celles qui s'imposent aux opérations sur bilan.

Le développement d'un « système bancaire parallèle » constitué de fonds divers a permis d'échapper aux règles de capital, puisque ces fonds n'étaient pas soumis à des contraintes réglementaires et de surveillances.

La détérioration des méthodes d'analyse et de gestion des risques. 
La politique monétaire très accommodante au risque i.e. La sanction des pertes en cas de récession était pratiquement éliminée par les banques centrales.

La supervision des banques était étonnement faible ces dernières années.

La généralisation de la garantie des dépôts par un fonds de garantie, dont toutes les banques et tous les établissements de crédit adhèrent; par cette pratique les gouvernements ont cherché à rassurer les déposants, mais aussi, à conforter la confiance de tous dans la stabilité du système bancaire.

L'aléa moral a eu un coût phénoménal lors de la crise bancaire et financière de l'automne 2008, qui s'est traduit par des montants énormes engagés par les Etats pour le renflouement des banques sous forme de: liquidité, recapitalisation, garanties diverses..., dans l'espoir de rassurer les déposants et d'endiguer un éventuelle «Bank Run ${ }^{7} »$.

Afin de pallier ce coût épouvantable et contenir l'aléa moral en matière financière, les instances nationales et internationales ont proposées de nombreuses mesures sous forme de directive pour le redressement et la résolution des défaillances bancaires.

Ce nouveau régime de résolution bancaire doit s'appliquer à toutes les institutions financières européennes, dont la défaillance pourrait se répercuter de manière importante sur l'ensemble du système financier.

\section{La résolution bancaire}

La résolution bancaire signifie l'intervention préventive d'une autorité publique sur un établissement bancaire ou financier avant la défaillance de celui-ci, de façon à le restructurer ou en opérer une liquidation ordonnée dans le but de limiter l'impact sur les clients de l'établissement et sur le reste de l'économie.

La directive sur le redressement et la résolution bancaire prévoit trois volets :

La prévention:

La prévention permet d'énoncer les mesures envisagées à prendre en cas de détérioration de la situation financière à travers des plans de redressement.

Pour les banques: elles devront élaborer des plans de redressement qui décrivent les mesures à prendre en cas de dégradation de leur situation financière afin de rétablir leur viabilité.

Pour les autorités nationales de résolution : elles devront élaborer des plans de résolution pour chaque établissement, avec des options pour gérer des banques qui sont dans une situation critique et ne peuvent plus être sauvées.

\section{L'intervention précoce :}

Dès lors qu'un établissement ne respecte plus ses exigences de fonds propres ou risque de ne plus les respecter, les autorités de surveillance pourront exiger de la banque la mise en œuvre de son plan de redressement, la réunion des actionnaires ainsi que la nomination d'un administrateur pour une durée limitée, si les instruments précédent s'avèrent insuffisants.

\section{La résolution :}

Celle-ci intervient, si les instruments précédents ne sont pas suffisants.

La directive prévoit quatre principaux instruments de résolution harmonisés :

La cession des activités (ou d'une partie des activités) à un acquéreur.

La création d'un établissement relais (bridge bank) sous contrôle public, afin d'y transférer temporairement des actifs bancaires sains.

La séparation d'actifs (transfert des actifs toxiques vers une structure de gestion des actifs : (SPV) ou (bad bank)).

Le renflouement interne (bail-in) qui préserve l'établissement tout en imposant les pertes aux créanciers.

Dans le cadre de la résolution bancaire, il fallait changer le paradigme du sauvetage bancaire, en passant du «bail-out» qui signifie : une recapitalisation par les gouvernements en 
mobilisant les fonds publics, au «bail-in » qui signifie: une recapitalisation par une mise à contribution des actionnaires et/ou des créanciers de la banque.

Par le passage du «bail-out» au «bail-in», il y a une volonté d'éviter les aléas de moralité, car avec le « bail-out» les :

Etats étaient incités à laisser croître excessivement leurs dettes publiques.

Banques étaient incitées à :

Prendre des risques excessifs.

Détenir des actifs risqués.

Accroître leur levier d'endettement.

Prêter à des emprunteurs risqués, d'où la hausse des prêts non performants.

Préteurs étaient certains d'être remboursés, de ce fait, ils étaient incités à ne pas surveiller les emprunteurs et ne plus exigés de prime de risque.

Quelles sont les modalités du bail-in?

Le bail-in impose à certain créanciers d'un établissement de crédit qui aurait des problèmes de solvabilité la conversion de leurs créances en actions de cet établissement et/ou la réduction du montant de ces créances.

Il s'agit donc d'un outil permettant de limiter les éventuels concours de fonds publics à un établissement en difficulté encore en activité ou en cours de liquidation (le bail-in permet de résoudre des problèmes de solvabilité mais pas de liquidité).

En cas d'insuffisance des capitaux propres suite à des pertes, ceux qui devront être sollicités pour le sauvetage de l'établissement, selon une hiérarchisation de mise à contribution du passif sont :

Actionnaires.

Dettes subordonnées.

Dettes seniors.

Dépôts non garantis des :

Grandes entreprises.

PME (Petites et Moyennes Entreprises).

BEI (la Banque Européenne d'Investissement).

Épargnants.

Par contre, ceux qui devront être exclus du passif sont :

Les dépôts garantis.

Les covered bonds ${ }^{8}$.

Les passifs liés aux activités commerciales de l'établissement.

La rémunération des employés de l'établissement.

Les passifs interbancaires d'une maturité inferieure à sept jours.

La flexibilité dont disposeront les autorités nationales dans l'application de ces règles restera restreinte (notamment dans l'exclusion au cas par cas de certains passifs) : elle ne pourra s'appliquer que lorsque les actionnaires auront été mis à contribution pour un montant équivalent à $8 \%$ du passif de l'établissement dont les fonds propres (ou $20 \%$ des actifs pondérés du risque, cas par cas). Parallèlement, les fonds de résolution ne pourront contribuer qu'à hauteur de $5 \%$ du passif de la banque.

Dans ce cadre, les autorités nationales pourront exclurent discrétionnairement certains passifs :

S'ils ne peuvent être mis à contribution dans une période raisonnable.

Pour permettre d'assurer la continuité des activités critiques de la banque.

Pour éviter les effets de contagion.

Ecarter le risque d'effet de valorisation pouvant accroître le montant global des pertes.

Ces exclusions devront être compensées soit par la contribution du fonds de résolution ou l'augmentation de la mise à contribution des autres passifs. 
Les différents instruments de résolution seront financés par des fonds de résolution nationaux, eux-mêmes abondés ex ante par un prélèvement sur le système bancaire (ce qui écarte la mobilisation de fonds publics dans le cadre de restructuration). La contribution des banques sera annuelle et calculée sur la base de leurs passifs respectifs.

Quel est le rôle des fonds de résolution des défaillances bancaires ? ${ }^{9}$

Les fonds de résolution doivent contribuer au financement de résolution ordonné des défaillances des banques en difficulté ; ils peuvent également être utilisés pour couvrir des coûts administratifs, juridiques et de conseil ; cependant, ils ne doivent pas jouer le rôle d'une assurance contre la faillite ou être utilisés pour renflouer des banques défaillantes.

Comment financer les fonds de résolution des défaillances bancaires ?

Trois éléments peuvent constituer l'assiette retenue pour le calcul de la contribution aux fonds de résolution :

Les actifs des banques peuvent constituer des indicateurs du montant nécessaire pour gérer une éventuelle défaillance de la banque. Un prélèvement peut être établi sur la base des actifs et se traduire par une exigence supplémentaire en matière de fonds propres.

Les passifs bancaires peuvent aussi constituer des indicateurs du montant nécessaire pour gérer une éventuelle défaillance de la banque. Cependant, les passifs, pourraient être des variables moins précises pour évaluer le degré de risque.

Les bénéfices et les primes peuvent servir de référence afin de déterminer le montant des prélèvements.

Les mécanismes de financement des fonds de résolution de défaillance bancaire doivent répondre aux critères suivants :

Eviter toute possibilité d'arbitrage.

Tenir compte des risques d'une manière appropriée.

Prendre en compte la nature systémique de certaines entités financières.

Se fonder sur le montant qui devrait être mobilisés si la résolution de défaillance devenait nécessaire.

Eviter les distorsions de concurrence.

\section{Conclusion et perspectives}

Il était question dans cet article d'étudier la pertinence des plans de gestion des crises bancaires élaborés au préalable par les autorités de réglementation et de surveillance, afin de réduire l'impact des défaillances bancaires et des institutions financières d'importance systémique, sur les contribuables qui lors de la crise financière de 2008 avaient été lourdement sollicités.

Le modèle dominant dans la résolution bancaire, semble être parfait pour gérer d'éventuelles crises futures, car il implique les créanciers d'une banque dans son sauvetage, en vue de les responsabiliser envers leurs décisions. Seulement, l'idée des créanciers inclus aussi les déposants, dans ce cas-là, combien de déposants savent vraiment que leur dépôt sont en réalité des prêts fait à leur banque et qu'ils comportent une part de risque ?

En effet, la plupart des déposants croient que l'argent déposé sur un compte bancaire est en sécurité dans ses coffres forts et assurément il reste leur propriété. En réalité, ces dépôts peu importe leur somme, sont considérés comme des prêts consentis à la banque, c'est pourquoi les déposants sont considérés - malgré eux- comme ses créanciers. Toutefois, nombreux pays ont fait en sorte que les petits déposants soient protégés, en bénéficiant d'une garantie implicite 
plafonnée de leur dépôt (seulement les dépôts inferieurs à 100.000 euros), de cette façon l'application du bail-in devient légitime.

Le dessein proclamé du «bail-in » est de préserver le contribuable des aberrations du système bancaire, tout en responsabilisant les gros déposants. En fait, ceux qui vont supporter les abus du système bancaire sont «le petit peuple » comme : les entreprises de bâtiment, les restaurants, les fermiers, les épiceries... et non ces oligarques qui seront toujours prévenus à temps afin qu'ils puissent retirer leurs fonds avant la saisie. En ce sens, le «bail-in » va contribuer à faire perdre le peu de confiance que les déposants ont envers le système bancaire, les poussant ainsi à retirer leur argent ce qui va anéantir l'activité principale des banques.

Ce modèle de gestion des crises bancaires pourra inciter, le temps aidant, les contribuables (la frontière est imperceptible avec les déposants) à préférer l'escamotage de leurs fonds, occasionnant la raréfaction des crédits, condition essentiel pour le financement de l'économie, cette situation va provoquer le dysfonctionnement du système financier, pour déboucher sur une crise systémique.

En conclusion, il faut échafauder un plan international capable de gérer des crises bancaires de dimension internationale. Cette idée est indéniable, en effet, il est primordial pour la robustesse et la stabilité de l'économie mondiale, que les régulateurs du système bancaire et financier de tous les pays qu'ils soient à puissance hégémonique ou en voie de développement de se réunir, afin de s'accorder sur le moyen d'éviter le déclenchement des crises. Toutefois, si une crise ne peut être contourné, il est essentiel qu'ils conviennent sur un procéder qui permet de contrecarrer la propagation d'une crise au reste du monde, en vue de, réduire les évolutions néfastes qui affectent la stabilité financière internationale.

Mais, quelles sont les méthodes efficientes?

Il est indispensable, en amont des difficultés, que les autorités de réglementation et de surveillance bancaire soient absolument impartiales, intransigeantes et irrémissibles envers toutes les banques du système bancaire mondial. Simultanément, les autorités doivent estimer la proportion de liberté indispensable à l'accomplissement de l'activité bancaire, cette juste mesure de l'autonomie consentie dépendra essentiellement de l'historique financier de chaque établissement bancaire.

Alors, à quand tous unis contre la malveillance du système bancaire ? 
Annexe 1

Tableau (1)

Les institutions financières d'importance systémique ${ }^{10}$

\begin{tabular}{|c|c|}
\hline \multirow{17}{*}{ Europe } & Barclays \\
\hline & BNP Paribas \\
\hline & BPCE (Banque Populaire Caisses d'Epargne). \\
\hline & Commezbank \\
\hline & Crédit Suisse \\
\hline & Deutsche Bank \\
\hline & Dexia \\
\hline & Groupe Crédit Agricole \\
\hline & HSBC(Hong Kong \& Shanghai Banking Corporation) \\
\hline & ING Bank(International Nederlanden Groep) \\
\hline & Lloyds Banking Group \\
\hline & Nordea \\
\hline & Royal Bank of Scotland \\
\hline & Santander \\
\hline & Société Générale \\
\hline & UBS \\
\hline & Unicredit Group \\
\hline \multirow{8}{*}{ Amérique } & Bank of America \\
\hline & Bank of New York Mellon \\
\hline & Citigroup \\
\hline & Goldman Sachs \\
\hline & JP Morgan Chase \\
\hline & Morgan Stanley \\
\hline & State Street \\
\hline & Wells Fargo \\
\hline \multirow{4}{*}{ Asie } & Bank of China \\
\hline & Mitsubishi UFJ FG \\
\hline & Mizuho FG \\
\hline & Sumitomo Mitsui FG \\
\hline
\end{tabular}


Bibliographiques

Comité de Bâle sur le contrôle bancaire. (2011). Banques d'importance systémique mondiale : méthodologie d'évaluation et exigence de capacité additionnelle d'absorption des pertes. Repéré à http://www.bis.org/publ/bcbs207_fr.pdf

De Larosière, J. (2011). Le hasard moral. Repéré à http://www.asmp.fr/travaux/communications/2011_03_14larosiere.htm

Dissaux, T. Lepetit, J-F. (2011). Prévention, traitement et résolution des crises bancaires. Revue d'économie financière, $\mathrm{n}^{\circ} 101$, 219-230. Repéré à http://www.cairn.info/resume.php?ID_ARTICLE=ECOFI_101_0219

Financial Stability Board. (2013). Update of group of global systemically important banks (G-SIBS). Repéré à http://www.financialstabilityboard.org/wpcontent/uploads/r_131111.pdf?page_moved $=1$

Fortésa, M-H. Kubrushko, A. (2012). Plan de redressement et de résolution : une nouvelle donne dans la gestion des crises bancaires. Revue Banque, $\mathrm{n}^{\circ} 308$. Repéré à http://www.revue-banque.fr/risques-reglementations/article/plans-redressement-resolution-unenouvelle-donne-d

Glossaire de termes des assurances et des financements ruraux. Organisation des Nations Unies et l'agriculture, Bulletin des services agricoles de la FAO 100. ISSN : 1014-4218, page 138.

Guersent, O. (2012). Résolution des crises bancaires : vers un cadre commun européen. Revue Banque, $\mathrm{n}^{\circ} 753$. Repéré à http://www.revue-banque.fr/risquesreglementations/article/resolution-des-crises-bancaires-vers-un-cadre-comm

Lemangnen, A. (2013). From bail-out to bail-in: the paradigm shift is official. Wholesale Banking, $\mathrm{n}^{\circ} 121$.

Rapport FMI/BRI/CSF. (2009). Guidance to assess the systemic importance of financial institutions, markets and instrument: initial consideration. Repéré à http://www.imf.org/external/np/g2df/100109.pdf

Thomson, James B. (2010). On Systemically Important Financial Institutions and Progressive Systemic Mitigation. Federal Reserve Bank of Cleveland. Repéré à $\mathrm{http} / / /$ works.bepress.com/cgi/viewcontent.cgi?article=1000\&context=james_thomson

http://europa.eu/legislation summaries/internal market/single market services/financial services_banking/mi004_fr.htm

http://fr.wikipedia.org/wiki/Dodd-

Frank_Wall_Street_Reform_and_Consummer_Protection_Act

\section{Références}

${ }^{1}$-La CRA a pour objet premier de contrer le refus des banques de prêter aux personnes dont le revenu faible ou modéré.

${ }^{2}$-Le prix de l'immobilier aux États-Unis n'a pas connu de baisse depuis la seconde guerre mondiale.

${ }^{3}$-Dodd-Frank Act est une loi pour promouvoir la stabilité financière des États-Unis en améliorant la responsabilisation (accountability) et la transparence dans le système financier, pour mettre fin au « too big to fail », pour protéger le contribuable américain en mettant fin au sauvetage financier (ending bail-out), pour protéger le consommateur des pratiques de services financiers abusifs et pour d'autres objectifs. 
La loi est divisée en 16 titres et couvre 848 pages, son nom provient de ses inspirateurs, le député Barney Frank et le sénateur Chris Dodd.

4-Rapport FMI/BRI/CSF. Guidance to assess the systemic importance of financial institutions, markets and instrument: initial consideration, October 2009.

5-Comité de Bâle sur le contrôle bancaire. Banques d'importance systémique mondiale : méthodologie d'évaluation et exigence de capacité additionnelle d'absorption des pertes. Texte des règles, Novembre 2011.

${ }^{6}$-Glossaire de termes des assurances et des financements ruraux. Organisation des Nations Unies et l'agriculture, Bulletin des services agricoles de la FAO 100. ISSN : 1014-4218, page 138.

7-Le « bank run » est l'affluence massive de clients dans les banques afin de retirer leurs économies, cette affluence intervient suite à l'annonce d'une faillite d'une banque.

${ }^{8}$-Les covered bonds sont des titres de dette senior à faible risque.

9 -

http://europa.eu/legislation_summaries/internal_market/single_market_services/financial_service s_banking/mi004_fr.htm-

${ }^{10}$-Financial Stability Board. Update of group of global systemically important banks (G-SIBS). 1 November 2012 